\title{
Dissociation of implicit memory in dementia: Neurological implications
}

\author{
NELSON BUTTERS \\ Veterans Administration Medical Center, San Diego, California \\ and University of California, San Diego, La Jolla, California \\ and \\ WILLIAM C. HEINDEL and DAVID P. SALMON \\ University of California, San Diego, La Jolla, California
}

\begin{abstract}
Lexical and pictorial priming tasks as well as tests of motor skill learning and weight biasing were administered to patients in the early stages of Alzheimer's Disease (AD) and Huntington's Disease (HD) and to intact control subjects. Lexical priming was evaluated with a stem-completion and a paired-associate paradigm, and perceptual priming was measured with a task involving the identification of fragmented drawings of common objects. A pursuit-rotor task was used to assess motor skill learning. Although both patient groups were severely impaired on explicit memory tasks, they exhibited marked dissociations on the implicit tests. The AD patients showed little lexical and pictorial priming, but performed like the control subjects on the weight-biasing and pursuit-rotor tasks. In contrast, the HD patients were unable to acquire the pursuit-rotor skill and demonstrated little weight biasing while performing like the control subjects on the lexical and pictorial priming tasks. These findings indicate that different forms of implicit memory may depend upon distinct constellations of brain structures. The integrity of the association cortices affected in $\mathrm{AD}$ may be necessary for the maintenance of lexical and pictorial priming, whereas the basal ganglia damaged in HD may be critical for the development of the motor programs underlying skill learning and weight biasing.
\end{abstract}

Although the specific neurological entities underlying explicit memory have been extensively described (Butters \& Miliotis, 1985; Squire, 1986), little attention has been given to the brain structures responsible for various forms of implicit memory. Damage to the mesial portions of the temporal lobes (especially the hippocampi), the medial diencephalon, and the basal forebrain all result in severe impairments on both verbal and nonverbal explicit memory tasks (for reviews, see Butters \& Stuss, 1989; Salmon \& Butters, 1987; Squire, 1987). In contrast, little can be stated with any certainty about the brain structures that mediate various forms of verbal and pictorial priming, skill learning, and classical conditioning. Some evidence exists linking the cerebellum to classical conditioning (Thompson, Berger, \& Madden, 1983), but only vague speculations have been offered for other forms of implicit memory.

To gather some initial clues concerning possible neurological dissociations among various forms of implicit memory, we have been evaluating the performances of patients with Alzheimer's Disease (AD) and with Huntington's Disease (HD) on verbal and pictorial priming,

This study was supported in part by funds from the Medical Research Service of the Veterans Administration, and by NIA Grants AG-05131 and AG-08204 to the University of California at San Diego. Address all correspondence to Nelson Butters, Psychology Service (116B), VA Medical Center, 3350 La Jolla Village Drive, La Jolla, CA 92161. skill learning, and weight-biasing tasks. Although there is merit to the old adage that such dementias ultimately result in diffuse damage to numerous cortical and subcortical systems, there is considerable evidence that in the early stages of these disorders, patients manifest quite different patterns of brain dysfunction. The pathological changes associated with early AD are focused primarily in cortical association regions, especially the mesial and lateral surfaces of temporoparietal regions (Brun, 1983; Terry \& Katzman, 1983). For HD, which is characterized by involuntary choreiform movements as well as a progressive dementia, the early brain changes involve the basal ganglia, especially the head of the caudate nucleus (Bruyn, Bots, \& Dom, 1979). Thus, in its earliest stages, AD appears to be primarily a "cortical" dementia, and HD a "subcortical" or basal ganglia disorder (Cummings \& Benson, 1984).

The main point of this review will be that $\mathrm{AD}$ and $\mathrm{HD}$ patients, in the early stages of their disorders and matched for overall degree of cognitive loss, can be dissociated with implicit memory tasks that involve the priming of semantic memory and the initiation of motor programs. That is, although patients with $\mathrm{AD}$ are impaired on verbal and pictorial priming tasks, HD patients fail to acquire motor skills and to demonstrate weight-biasing effects. This double dissociation suggests that portions of the association cortex and the basal ganglia mediate quite different forms of implicit memory: the association cor- 
tices appear to be vital to tasks that rely upon the integrity of semantic knowledge, the basal ganglia to tasks that involve the generation of motor programs to guide behavior.

\section{Lexical and Pictorial Priming}

In two studies (Salmon, Shimamura, Butters, \& Smith, 1988; Shimamura, Salmon, Squire, \& Butters, 1987), $A D, H D$, and amnesic patients were compared with normal controls on lexical priming tasks using stem-completion and paired-associate paradigms. The stem-completion procedures were similar to those used by Graf, Squire, and Mandler (1984) in their demonstration of normal priming in amnesic patients. Briefly, subjects were shown 10 words (e.g., MOTEL, ABSTAIN) one at a time and were asked to rate how much they liked each word on a 5-point scale. In Salmon et al.'s (1988) study, this rating procedure was carried out twice, with the words presented in the same order on both occasions. Following these two presentation trials, the subjects were shown 20 three-letter word stems (e.g., MOT, ABS) and were asked to complete each stem with the first word that came to mind. Ten of the stems could be completed using study words, and the other 10 stems were used to assess baseline guessing rates. After two administrations of this stem-completion procedure, the subjects' performances on explicit recall and recognition of 10 -word lists were evaluated.

The results of both studies (Salmon et al., 1988; Shimamura et al., 1987) were similar, and those of Salmon et al. (1988) are shown in Figures 1 and 2. As anticipated, the $\mathrm{AD}, \mathrm{HD}$, and amnesic (alcoholic Korsakoff) patients were impaired relative to their agematched intact controls on both the explicit recall and the recognition memory tests (Figure 1 ). However, major differences among the three patient groups were found on the stem-completion task (Figure 2). Although baseline guessing rates (shaded portions) did not differ across the groups, the AD patients showed significantly less stem-completion priming than did the HD and amnesic patients and the intact controls. That is, relative to the other subjects, the $\mathrm{AD}$ patients were less likely to complete the stems with one of the previously presented words. The HD and amnesic patients were not able to recall or recognize many of the presented words when tested under explicit conditions, but they did produce these words when their memory was tested implicitly with the stemcompletion paradigm.

Salmon et al. (1988) reported a very severe semantic priming deficit in AD patients with a paired-associate procedure. $\mathrm{AD}, \mathrm{HD}$, and intact control subjects were asked to judge categorically or functionally related word pairs (e.g., BIRD-ROBIN, NEEDLE-THREAD) and later to say the first word that came to mind when presented with the first word (e.g., BIRD, NEEDLE) of a pair. It was proposed that the subjects' tendencies to produce the second word of the functionally or categorically related pairs would serve as a measure of the intactness of semantic priming and of the organization of semantic knowledge. As seen in Figure 3, the HD patients
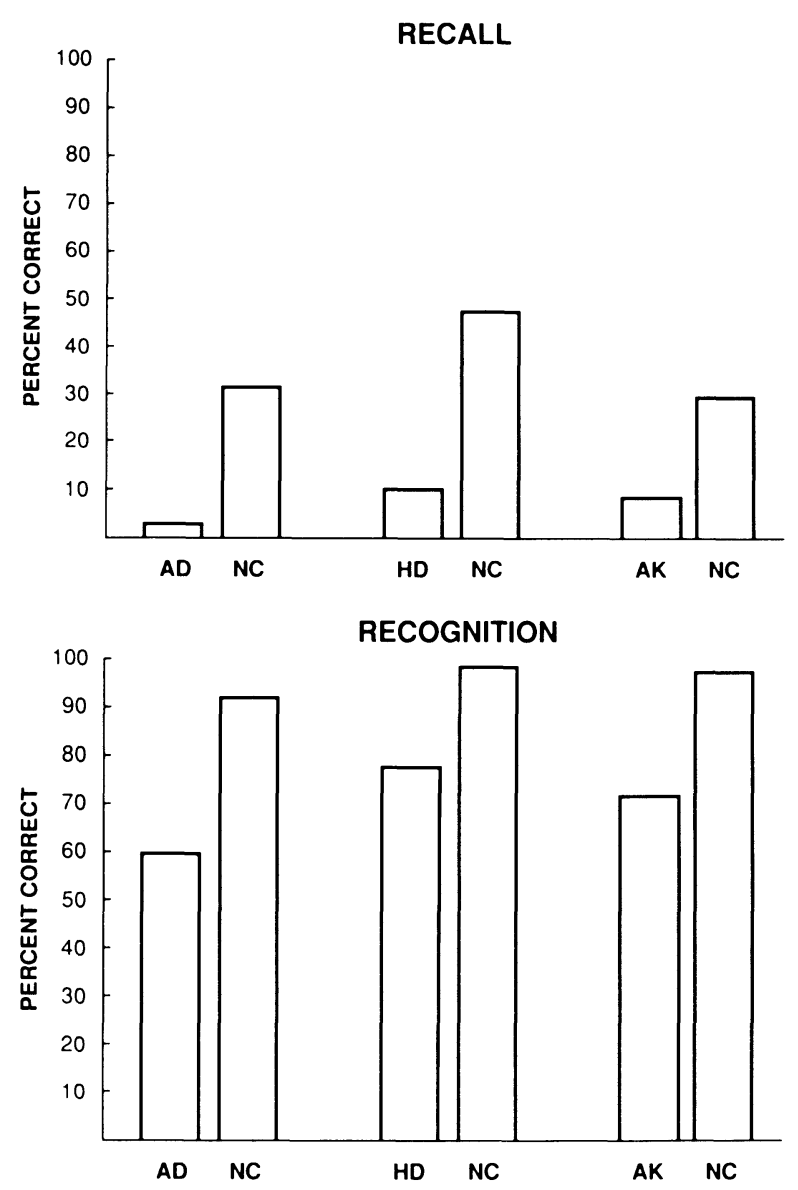

Figure 1. Percentage of words correctly recalled (top graph) or recognized (bottom graph) in the lexical priming task by Alzheimer's Disease (AD), Huntington's Disease (HD), and alcoholic Korsakoff (AK) patients, and their age-matched normal control (NC) subjects. (Modified from Salmon et al., 1988.)

demonstrated normal semantic priming on this task, and performed significantly better than the AD patients. The AD group was significantly impaired relative to their control group, and was the only group that did not respond to priming above baseline guessing rates. The same findings were apparent for both categorically and functionally related items.

The results of this paired-associate priming study suggest that the noted failures in $\mathrm{AD}$ patients' lexical priming may be due to some partial breakdown in the associative structure of semantic memory. The categorical and functional cues may have failed to activate traces of previously presented stimuli due to the dissolution of the semantic network governing verbal materials. For example, the cue BIRD may not have evoked an unconscious activation of the categorical associate ROBIN because the association between the two words had been greatly weakened. Such a disruption of the organization of semantic memory would also account for the AD patients' impairment on the stem-completion task. The association in semantic memory between a word stem such as MOT and 


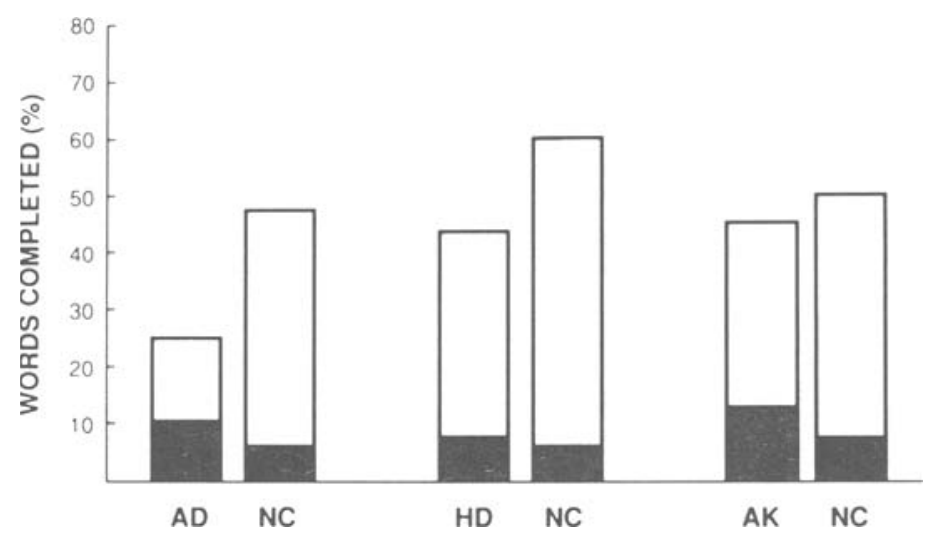

Figure 2. Percentage of word stems completed with previously presented words in the lexical priming task by Alzheimer's Disease (AD), Huntington's Disease (HD), and alcoholic Korsakoff (AK) patients, and their age-matched normal control (NC) subjects. The shaded portion of each bar indicates the baseline guessing rate for each subject group. (Modified from Salmon et al., 1988.)

the word MOTEL may have been sufficiently disrupted to negate the facilitating effect of the word's presentation.

Further evidence that $\mathrm{AD}$ and $\mathrm{HD}$ patients can be dissociated with priming tasks that engage or are dependent upon semantic associations has been found with a picturepriming task (Heindel, Salmon, \& Butters, in press). Facilitation effects with pictorial stimuli have previously been demonstrated in both normal subjects and amnesic patients (Warrington \& Weiskrantz, 1968). Although some studies (see Kosslyn, 1980; Paivio, 1983) suggest that pictures and words are represented by qualitatively distinct semantic codes, other investigations (Bajo, 1988; Vanderwart, 1984) have demonstrated strong cross-form facilitation when both words and pictures are processed at a semantic level. To the extent that words and pictures are indeed represented by a single, shared semantic representation (Anderson \& Bower, 1974; Nelson, Reed, \& McEvoy, 1977), one would expect AD patients to be impaired in the priming of both types of stimuli. On the other hand, if HD patients' semantic memory remains structurally intact, they should demonstrate normal levels of facilitation with pictorial as well as with lexical materials.

Heindel et al. (in press) designed their study to be directly comparable to the stem-completion test of verbal priming (Graf et al., 1984; Salmon et al., 1988; Shimamura et al., 1987). AD, HD, and intact agematched control subjects were first shown 30 completed, realistic line drawings of common objects (e.g., tree, bicycle) and were simply asked to name the object depicted. All subject groups performed well (mean between 29 and 30 correct) on this naming task. Ten minutes following

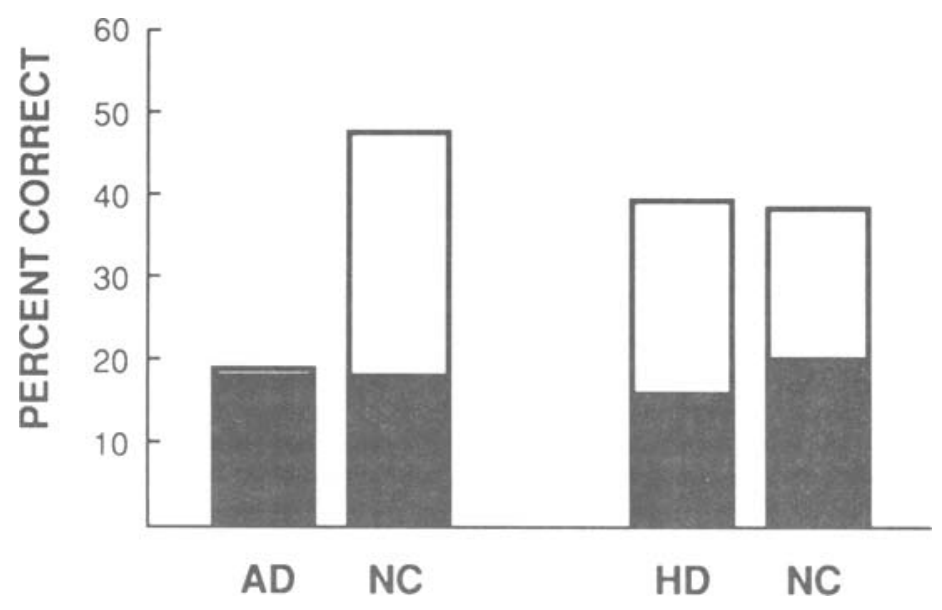

Figure 3. Percentage of previously presented words correctly produced in the free-association task by Alzheimer's Disease (AD) and Huntington's Disease (HD) patients, and their age-matched normal control subjects (NC). The shaded portion of each graph indicates the baseline guessing rate for each subject group. (Modified from Salmon et al., 1988.) 
the naming test, the fragment-identification task was administered. The examiner showed the subjects fragmented versions $(50 \%, 60 \%, 70 \%$, or $80 \%$ of the picture eliminated) of 15 of the 30 objects from the naming test and of 15 distractor pictures that had not been presented previously. The subjects were asked to look at each fragmented drawing and to relate as quickly as possible the first thing they thought the picture might be (see Schacter, Cooper, \& Delaney, 1990, for a similar task). The examiner encouraged guessing and was careful not to associate this "perceptual" test with the "naming" task that had preceded it. The subjects were always shown the most (80\%) fragmented version of each picture first and the least $(50 \%)$ fragmented version last. Testing continued until the subject correctly identified all 30 items (i.e., 15 implicit targets, 15 distractors) on a single trial, or until the subject completed the final (fifth) trial, in which the complete version of the figures were presented. Immediately after completion of the fragment-identification test, the subjects were given an explicit cued-recall test using fragments of the 15 remaining pictures exposed during the naming task. The subjects were told that the fragments represented some of the pictures they had named previously and that they were to try to remember what they were. Again, the most fragmented (80\%) pictures were shown first and the least fragmented $(50 \%)$ last. Testing continued until the subject correctly identified all 15 items on a single trial.

Two measures were calculated to assess the subjects' performances: naming thresholds and facilitation scores. A naming threshold was calculated separately for the 15 implicit targets and the 15 distractors from the identification test, and for the 15 explicit targets from the cuedrecall test. The naming threshold score ranged from 1 (the subject named a target or distractor at the most fragmented level) to 5 (the subject named a target or distractor only when it was completely drawn). Facilitation scores were calculated using the naming thresholds for the 15 implicit targets and the 15 distractors on the identification test. For each subject, the actual difference between mean naming thresholds for implicit targets and distractors was divided by the possible reduction in naming thresholds. For example, if a subject obtained a mean naming threshold of 2.5 on the distractor items and reduced his or her naming threshold to 1.9 on target items, then that subject showed an actual facilitation of 0.6 (i.e., $2.5-$ 1.9) out of a possible facilitation of 1.5 (i.e., $2.5-1.0$ ).
Thus, this subject's facilitation score would be 0.40 (or $40 \%)$.

Table 1 shows the performances of $\mathrm{AD}, \mathrm{HD}$, and intact age-matched control subjects on this pictorial priming task. Both intact control groups and the HD group earned significantly lower scores (i.e., more rapid identification) on implicit targets than on distractors, whereas the AD patients evidenced no significant difference on these two measures. The facilitation scores also revealed this difference between $A D$ and HD patients on pictorial priming. The $\mathrm{AD}$ patients had significantly lower facilitation scores than the other three subject groups, whereas the HD patients' scores did not differ from those of the two control groups. As was expected of demented patients with severe explicit memory deficits, both the AD and the HD patients had significantly higher naming thresholds for explicit targets than did their corresponding agematched controls.

\section{Skill Learning and Weight Biasing}

Although the striking and consistent impairments of $\mathrm{AD}$ patients on lexical and pictorial priming tasks suggest that these forms of implicit memory may be dependent upon the intactness of association cortices (especially the temporoparietal regions that underlie language functions and the structure of semantic knowledge), another series of studies have pointed to the basal ganglia as the critical neurological structures for the acquisition of skill-based knowledge and other implicit tasks requiring the generation of motor programs to guide behavior. Stimulated by a report (Martone, Butters, Payne, Becker, \& Sax, 1984) that HD patients are impaired in the acquisition of a mirror-reading skill, Heindel and his colleagues (Heindel, Butters, \& Salmon, 1988; Heindel, Salmon, Shults, Walicke, \& Butters, 1989) evaluated AD, HD, and amnesic patients and intact control subjects on a pursuit-rotor task. This classical test of skill learning has the advantage that it avoids some of the methodological problems encountered by Martone et al. on the mirror-reading test. For example, the HD patients' impairments in reading mirror-reflected words may have been more attributable to the eye-movement problems associated with this disease than to a general impairment in skill learning. In addition, the interpretation of Martone et al.'s results were further confounded by the failure of the HD patients to begin the mirror-reading task at the same initial level of performance as the other subjects (i.e., they were slower

Table 1

Performance of Alzheimer's (AD) and Huntington's (HD) Disease Patients and Elderly (ENC) and Middle-Aged (MNC) Normal Control Subjects on the Pictorial Priming Task

\begin{tabular}{lcccc}
\hline Subjects & $\begin{array}{c}\text { Naming Threshold } \\
\text { Implicit Targets }\end{array}$ & $\begin{array}{c}\text { Naming Threshold } \\
\text { Distractors }\end{array}$ & $\begin{array}{c}\text { Facilitation } \\
\text { Score (\%) }\end{array}$ & $\begin{array}{c}\text { Naming Threshold } \\
\text { Explicit Targets }\end{array}$ \\
\hline AD & 2.16 & 2.37 & 13.2 & 2.13 \\
HD & 1.62 & 2.69 & 63.5 & 1.80 \\
ENC & 1.57 & 2.16 & 52.1 & 1.40 \\
MNC & 1.42 & 1.99 & 57.9 & 1.24 \\
\hline
\end{tabular}


than amnesic patients and intact control subjects). Different levels of initial performance can result in ceiling or floor effects that reduce the meaningfulness of the results. In Heindel et al.'s investigations, initial performance levels were equated by manipulating the difficulty (i.e., speed of rotation of the disk) of the pursuit-rotor task.

The subjects were told to try to maintain contact between a stylus held in the preferred hand and a small metallic disk ( $2 \mathrm{~cm}$ in diameter) on a rotating turntable ( $25 \mathrm{~cm}$ in diameter). The turntable could be adjusted to rotate at $15,30,45$, or 60 rotations per minute for a given 20 -sec trial. All subjects were tested over three sessions of eight trials each, with each session separated by approximately $30 \mathrm{~min}$ of other psychometric testing. Within each test session, the subjects were allowed a 1-min rest interval between the fourth and fifth trials, thereby creating six blocks of four trials each. The total time on target was recorded for each 20 -sec trial. On the basis of preliminary practice trials, the turntable was set for each subject at a particular speed that would ensure that he or she would commence testing with a score close to $25 \%$ (i.e., $5 \mathrm{sec}$ ) on target. In this manner, initial level of performance on the pursuit-rotor task was equated for the subject groups.

The results (Heindel et al., 1988; Heindel et al., 1989) showed that the HD patients were severely impaired in the acquisition of the pursuit-rotor skill (Figure 4). Although the $\mathrm{AD}$ and amnesic patients and the intact control subjects all demonstrated systematic and equivalent improvement in performance over the six blocks of trials, the HD patients evidenced only slight improvements during the entire training session. Furthermore, it was found

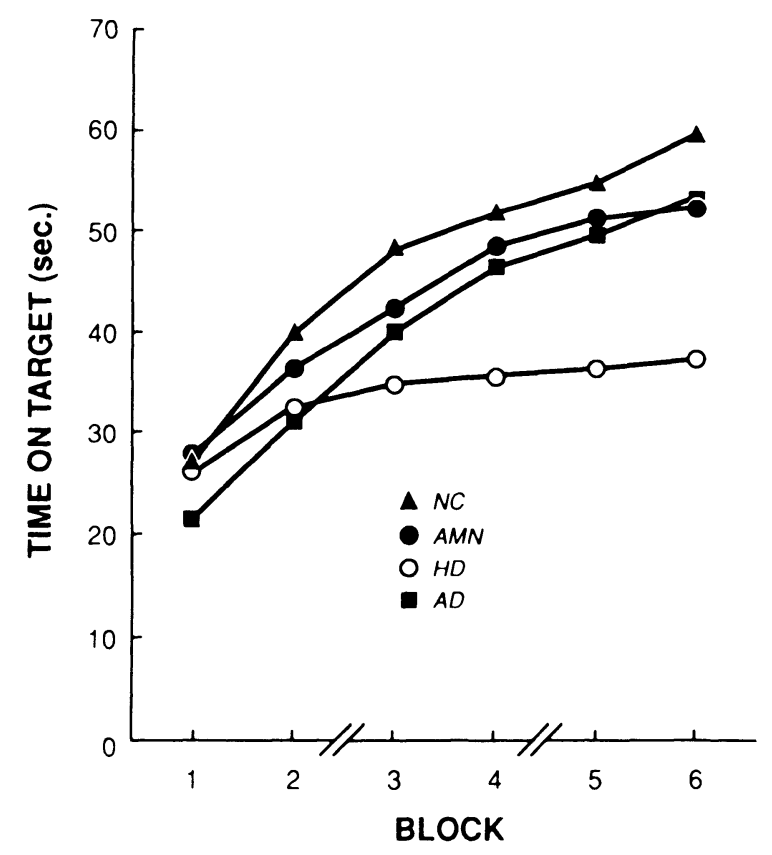

Figure 4. Performance of Alzheimer's Disease (AD), Huntington's Disease (HD), and amnesic (AMN) patients, and normal control (NC) subjects on the pursuit-rotor task. (Modified from Heindel et al., 1988.) that the HD patients' performance was not associated with either their overall level of functional disability (Heindel et al., 1988) or their degree of chorea and other extrapyramidal motor signs (i.e., tremor, rigidity, bradykinesia; Heindel et al., 1989). Since only severity of dementia was significantly correlated $(r=.58, p<.05)$ with the HD patients' deficits on pursuit-rotor learning, Heindel et al. (1989) concluded that their impairment in skill learning was a feature of their cognitive losses and not a consequence of some basic motor disability.

In addition to the pursuit-rotor task, Heindel et al. (1989) also administered the stem-completion priming task described previously in this paper. Like the previous studies (Salmon et al., 1988; Shimamura et al., 1987), Heindel et al. found that the AD patients were significantly less likely than the HD patients and intact control subjects to manifest stem-completion priming. Thus, within a single investigation, using the same subjects, a double dissociation between skill learning and lexical priming in $\mathrm{HD}$ and $\mathrm{AD}$ patients was noted. It appears then that the association cortices play an important role in lexical priming, whereas the basal ganglia seem to be critical for the learning of motor and visuomotor skills. The reports that HD patients are impaired in learning the solution of complex problem-solving tasks suggest that cognitive skill learning also may be at least partially dependent upon the intactness of the basal ganglia (Butters, Wolfe, Martone, Granholm, \& Cermak, 1985; Saint-Cyr, Taylor, \& Lang, 1988).

To determine more directly whether HD patients' impairments on the pursuit-rotor task are related to some deficiency in the initiation of motor programs, Heindel, Salmon, and Butters (in press) administered a form of Helson's (1948) classic weight-biasing task to demented and control subjects. Since weight judgments are believed to involve motor programs and expectancies and do not require complex motor movements for their execution, this psychological task seemed ideal for use with HD patients, whose performance on motor skill learning tasks can easily be confounded by their basic extrapyramidal motor deficits. Thus, a demonstration that HD patients are less likely than $A D$ patients to manifest normal weight biasing would further strengthen the probability that their deficits on the pursuit-rotor test are due to a failure in skill acquisition, and not to some basic motor weakness and/or dysfunction.

The stimuli for this study were two sets (one brown and one gray) of 10 cylindrical-shaped weights ranging from 35 to $485 \mathrm{~g}$, in $50-\mathrm{g}$ increments. The test procedures were similar to those employed by Benzing and Squire (1989) with amnesic patients. During the biasing trials, the subjects were instructed to lift (with thumb and index finger) a series of weights and to judge whether each successive weight was heavier or lighter than the one that had just preceded it. The subjects were given 40 trials of either the five lightest weights (i.e., the light bias condition) or the five heaviest weights (i.e., the heavy bias condition). For each bias condition, the five weights were presented 
eight times each in a fixed random order. Twenty to 25 min after completing the biasing trials, a 7 -item recall and recognition test was administered to evaluate the subjects' explicit memory of the biasing trials.

Immediately following the explicit memory test, the subjects were administered 10 test (weight-judgment) trials. The subjects were told to lift and then judge each weight according to a 9-point rating scale (i.e., $1=e x$ tremely light; $9=$ extremely heavy). The subjects judged each of the 10 weights comprising a set in a fixed random order.

The subjects were administered a second set of 40 bias trials and 10 test trials approximately $2-3 \mathrm{~h}$ after the first bias condition. The order of conditions was counterbalanced across subjects so that half of the subjects within each group received the heavy bias condition first and the light bias condition second, and the other half received the light bias condition first and the heavy condition second. A different set of weights was used on the two weight-bias test conditions. A weight-biasing effect would be reflected in the subjects' tendency to rate the 10 standard weights as relatively heavier following the light bias condition and relatively lighter following the heavy bias condition (see Figure 5).

The performances of the AD, HD, and intact subjects did not differ in the weight-biasing conditions. All groups exceeded $90 \%$ accuracy in their judgment as to whether a given weight was lighter or heavier than the immediately preceding one and performed slightly better in the light bias condition than in the heavy bias condition. Thus, there was no indication in the bias conditions that any group had a sensory or motor deficit that significantly limited their ability to make weight judgments.

On the 10 test (weight-judgment) trials, however, group differences were noted. The AD patients and the intact control subjects evidenced the expected biasing effects: these subjects judged weights heavier after a light bias condition than after a heavy bias condition. That is, experiences limited to the five heaviest or lightest weights (i.e., bias conditions) led to biases and expectancies that influenced the $\mathrm{AD}$ and control subjects' judgments of the entire 10-weight set. In contrast to the AD patients and control subjects, the HD patients' ratings of the 10 weights did not differ significantly for the light and heavy bias conditions. Furthermore, for the HD patients, Heindel et al. (in press) found a significant correlation $(r=.57$, $p<.05$ ) between severity of dementia (as measured by scores on a mental status exam) and failure to show weight biasing. Since the correlations between severity of motor dysfunction and lack of weight biasing did not approach significance, Heindel et al. concluded that the HD patients' failures in weight biasing, like their deficiencies on pursuit-rotor learning, are associated with their dementia and not with their basic motor disturbances.

In view of the significant correlations with dementia, Heindel et al. (in press) divided both the HD and the AD patients into mild and moderately demented groups, as shown in Table 2 . Both mild and moderately demented $\mathrm{AD}$ patients demonstrated weight-biasing effects, whereas only the mildly demented HD patients exhibited the same

\section{Light Bias}

\section{Heavy Bias}

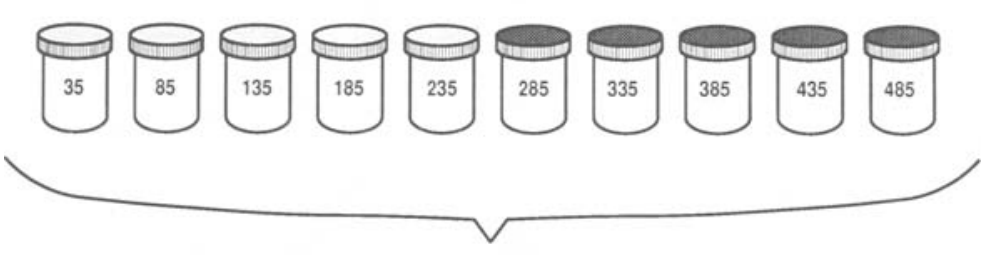

Perceived Heaviness of 10 Weights

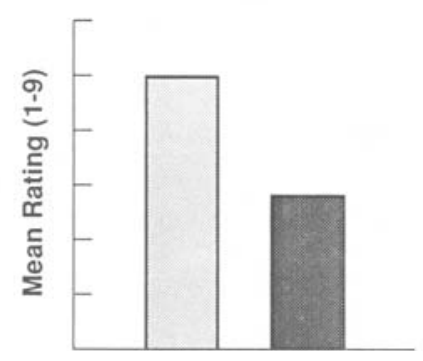

Figure 5. Schematic diagram illustrating the weight-biasing effect. Subjects tend to perceive the 10 standard weights as relatively heavier when they are initially exposed to the 5 lightest weights (light shading), and as relatively lighter when they are initially exposed to the 5 heaviest weights (dark shading). 
Table 2

Mean Weight Judgments by Mildly (MI) and Moderately (MO) Demented Huntington's (HD) and Alzheimer's (AD) Disease Patients after Light and Heavy Bias Conditions

\begin{tabular}{lcc}
\hline & \multicolumn{2}{c}{ Condition } \\
\cline { 2 - 3 } Subjects & Light Bias & Heavy Bias \\
\hline MI-HD & 6.3 & 5.4 \\
MO-HD & 5.6 & 5.8 \\
MI-AD & 6.2 & 5.4 \\
MO-AD & 6.0 & 5.3 \\
\hline
\end{tabular}

effect. Moderately demented HD patients showed no evidence that their ratings of the 10 weights were influenced by the biasing trials. It should also be noted that both the $A D$ and the HD patients were significantly impaired on the explicit memory test concerned with the recall and recognition of details pertaining to the weight-biasing task. Thus, many patients, especially those with $\mathrm{AD}$, who could not consciously remember the biasing task showed intact implicit memories for these biasing experiences when asked to judge the 10 test weights. Benzing and Squire (1989) have reported similar findings for amnesic patients.

When these results for the weight-biasing task are considered in conjunction with those for the pursuit-rotor test, several conclusions emerge. First, the HD patients' deficits likely reflect an inability to generate the central motor programs (or expectancies) that underlie both skill learning and some aspects of weight perception. Second, given the significant correlations between the HD patients' impairments and the severity of their dementia, it is unlikely that their poor performances can be attributed to basic motor dysfunctions. Deficiencies in the production of motor programs seem to be a central feature of these HD patients' cognitive losses. Third, the basal ganglia, especially the caudate nucleus and corticostriatal tracts, are critical for the production and retention of these motor programs.

\section{Summary and General Conclusions}

The studies reviewed in this paper indicate that patients with dementias of different etiologies and sites of neuropathology can also be differentiated by their performance on tests of implicit memory. Alzheimer patients were found to be severely impaired on lexical and pictorial priming tests, whereas HD patients demonstrated normal verbal and pictorial priming ability (Heindel et al., in press; Salmon et al., 1988; Shimamura et al., 1987). In contrast, HD patients were impaired on motor skill learning and weight-biasing tasks that were performed normally by $\mathrm{AD}$ patients (Heindel et al., 1988; Heindel et al., in press; Heindel et al., 1989). These differences in implicit memory performance occurred despite equivalent levels of overall cognitive dysfunction in the $\mathrm{AD}$ and $\mathrm{HD}$ patients.

These double dissociations between $\mathrm{AD}$ and $\mathrm{HD}$ patients on lexical and pictorial priming, skill learning, and weight- biasing tasks suggest that different forms of implicit memory, all of which are intact in patients with organic amnesic syndromes, are not mediated by a single neurological substrate. Rather, it appears that there are at least two psychologically and neurologically distinct implicit memory systems. Verbal and pictorial priming may both involve the temporary activation of stored representations in semantic memory, and may be dependent upon the functional integrity of the neocortical association areas damaged in AD. Motor skill learning and the biasing of weight perception, on the other hand, may both involve the modification of programmed movement parameters, and may be mediated by a corticostriatal system that is severely compromised in HD.

The results of these studies are consistent with the notion that memory is not a unitary entity but instead consists of a number of distinct yet interacting implicit and explicit memory systems (Schacter, in press; Tulving \& Schacter, 1990), and that different forms of implicit memory may involve changes in the processing structures that are involved in performing each particular task (Squire, 1987). If this view is correct, then future investigations with demented and other neurological populations may lead to the identification of additional implicit memory systems.

\section{REFERENCES}

ANDERSON, J. R., \& Bower, G. H. (1974). A propositional theory of recognition memory. Memory \& Cognition, 2, 406-412.

BAJO, M.-T. (1988). Semantic facilitation with pictures. Journal of Experimental Psychology: Learning, Memory, \& Cognition, 14, 579-589.

Benzing, W. C., \& Souire, L. R. (1989). Preserved learning and memory in amnesia: Intact adaptation-level effects and learning of stereoscopic depth. Behavioral Neuroscience, 103, 538-547.

Brun, A. (1983). An overview of light and electron microscopic changes. In B. Reisberg (Ed.), Alzheimer's disease (pp. 37-47). New York: Free Press.

BruYN, G. W., Bots, G., \& DoM, R. (1979). Huntington's chorea: Current neuropathological status. In T. Chase, N. Wexler, \& A. Barbeau (Eds.), Advances in neurology: Vol. 23. Huntington's disease (pp. 83-94). New York: Raven.

Butters, N., \& Miliotis, P. (1985). Amnesic disorders. In K. Heilman \& E. Valenstein (Eds.), Clinical neuropsychology (2nd ed., pp. 403-451). New York: Oxford University Press.

ButTERS, N., \& Stuss, D. T. (1989). Diencephalic amnesia. In F. Boller \& J. Grafman (Eds.), Handbook of neuropsychology (Vol. 3, pp. 107 148). New York: Elsevier.

Butters, N., Wolfe, J., Martone, M., Granholm, E., \& Cermak, L. S. (1985). Memory disorders associated with Huntington's disease: Verbal recall, verbal recognition and procedural memory. Neuropsychologia, 6, 729-744.

Cummings, J. L., \& Benson, F. (1984). Subcortical dementia: Review of an emerging concept. Archives of Neurology, 41, 874-879.

Graf, P., Squire, L. R., \& Mandler, G. (1984). The information that amnesic patients do not forget. Journal of Experimental Psychology: Learning, Memory, \& Cognition, 10, 164-178.

Heindel, W. C., Butters, N., \& Salmon, D. P. (1988). Impaired learning of a motor skill in patients with Huntington's disease. Behavioral Neuroscience, 102, 141-147.

Heindel, W. C., Salmon, D. P., \& Butters, N. (in press). The biasing of weight judgments in Alzheimer's and Huntington's disease: A priming or programming phenomenon? Journal of Clinical \& Experimental Neuropsychology. 
Heindel, W. C., Salmon, D. P., \& Butters, N. (in press). Pictorial priming and cued recall in Alzheimer's and Huntington's disease. Brain \& Cognition.

Heindel, W. C., Salmon, D. P., Shults, C. W., Walicke, P. A., \& BUTTERS, N. (1989). Neuropsychological evidence for multiple implicit memory systems: A comparison of Alzheimer's, Huntington's, and Parkinson's disease patients. Journal of Neuroscience, 9, 582-587.

Helson, H. (1948). Adaptation-level as a basis for a quantitative theory of frames of reference. Psychological Review, 55, 297-313.

Kosslyn, S. M. (1980). Image and mind. Cambridge, MA: Harvard University Press.

Martone, M., Butters, N., Payne, M., Becker, J., \& Sax, D. S. (1984). Dissociations between skill learning and verbal recognition in amnesia and dementia. Archives of Neurology, 41, 965-970.

Nelson, D. L., ReEd, V. S., \& McEvoy, C. L. (1977). Learning to order pictures and words: A model of sensory and semantic encoding. Journal of Experimental Psychology: Human Learning \& Memory, 3, 485-497.

Paivio, A. (1983). The empirical case for a dual coding. In J. C. Yuille (Ed.), Imagery, memory, and cognition: Essays in honor of Allan Paivio (pp. 307-332). New York: Erlbaum.

Saint-Cyr, J. A., TAYlor, A. E., \& Lang, A. E. (1988). Procedural learning and neostriatal dysfunction in man. Brain, 111, 941-959.

Salmon, D. P., \& Butters, N. (1987). The etiology and neuropathology of alcoholic Korsakoff's syndrome: Some evidence for the role of the basal forebrain. In M. Galanter (Ed.), Recent developments in alcoholism (Vol. 5, pp. 27-58). New York: Plenum.

Salmon, D. P., Shimamura, A. P., Butters, N., \& Smith, S. (1988).
Lexical and semantic priming deficits in patients with Alzheimer's disease. Journal of Clinical \& Experimental Neuropsychology, 10, $477-494$.

SChaCter, D. L. (in press). Perceptual representation systems and implicit memory systems: Toward a resolution of the multiple memory system debate. Annals of the New York Academy of Sciences.

SChaCter, D. L., CoOper, L. A., \& Delaney, S. M. (1990). Implicit memory for visual objects and the structural description system. Bulletin of the Psychonomic Society, 28, 367-372.

Shimamura, A. P., Salmon, D. P., SQuire, L. R., \& Butters, N. (1987). Memory dysfunction and word priming in dementia and amnesia. Behavioral Neuroscience, 101, 347-351.

SQuire, L. R. (1986). Mechanisms of memory. Science, 232, 1612-1619.

SQuiRE, L. R. (1987). Memory and brain. New York: Oxford University Press.

Terry, R. D., \& Katzman, R. (1983). Senile dementia of the Alzheimer type. Annals of Neurology, 14, 497-506.

Thompson, R. F., Berger, T. W., \& Madden, J. (1983). Cellular processes of learning and memory in the mammalian CNS. Annual Review of Neuroscience, 6, 447-491.

Tulving, E., \& SCHACTER, D. L. (1990). Priming and human memory systems. Science, 247, 301-306.

VANDERWART, M. (1984). Priming by pictures in lexical decision. Journal of Verbal Learning \& Verbal Behavior, 23, 67-83.

Warrington, E. K., \& Weiskrantz, L. (1968). New method for testing long-term retention with special reference to amnesic patients. $\mathrm{Na}$ ture, 217, 972-974. 